\title{
Toward Mid-Infrared Ultra-Short Pulse Generation in a Gas-Filled Hollow-Core Photonic Crystal fiber
}

\author{
Coralie Fourcade-Dutin ${ }^{1, *}$, Olivia Zurita Miranda ${ }^{1,2}$, Patrick Mounaix ${ }^{1}$ and Damien Bigourd ${ }^{1}$ \\ ${ }^{1}$ Laboratoire IMS, UMR CNRS 5218, University of Bordeaux, 33400 Talence, France \\ ${ }^{2}$ Institut FEMTO-ST, Département d'Optique, UMR CNRS 6174 -Université Bourgogne Franche-Comté, 25030 Besançon, France \\ *Correspondence: coralie.fourcade-dutin@u-bordeaux.fr;
}

\begin{abstract}
We numerically show that when a gas filled hollow-core photonic crystal fiber is pumped by two-color pulses, an ultra-short pulse can be generated in the mid-infrared. This phenomenon is due to the cascaded nonlinear phenomenon starting from a spectral broadening of the two pulses combined with an induced phase-matched four wave-mixing lying in the midinfrared, with a dispersive wave. By selecting the mid-infrared band with a bandpass filter, we demonstrate the generation of $60 \mathrm{fs}$ pulses at 3-4 $\mu \mathrm{m}$ and an ultra-short pulse duration of $20 \mathrm{fs}$ can be reached with additional phase compensator.
\end{abstract}

\section{Introduction}

These last years, an interest is growing for the mid-infrared (MIR) sources driven by demanding applications as gas sensing [1] or the creation of secondary sources [2]. An excellent method for the light generation in the MIR is to take benefit from nonlinear photonic devices to create down-converted frequencies from a pump laser. Here, we propose to generate ultra-short pulses in the MIR by pumping a gas-filled hollow-core photonic crystal fiber (HCPCF) with two different pulses to create an efficient phase-matched process. In addition, the frequency conversion from the pump to the MIR can be engineered with the dispersion of the waveguide and the gas contribution. It means that the phase matching can be tuned by changing the gas pressure. In our approach, only two ultra-short pump pulses are injected and the efficient phase matching is reached with the spectral broadening in the HC-PCF. The dynamics of the mechanism originated from cascaded nonlinear phenomenon including phase modulation and phase-matched four-wave mixing (FWM) processes. The FWMs are directly induced from the two broad-band pulses capable to generate pulses at 3 and $4 \mu \mathrm{m}$ with a pulse duration up to $20 \mathrm{fs}$.

\section{Principle and numerical methods}

In order to deeply understand the nonlinear pulse propagation and investigate the generation in the MIR, we conducted numerical simulations. The general principle of the process is to pump the HC-PCF with two different ultra-short pulses, $U P_{1}$ and $U P_{2}$, with spectra centered at $1030 \mathrm{~nm}$ and $515 \mathrm{~nm}$ corresponding to the emission of an ultra-fast ytterbium doped fiber laser and a second harmonic generator. The two pulses lie in the normal dispersion regime and the spectra broaden during the propagation in the fiber. Then, the MIR light is generated from a phase matched FWM between several created spectral components. The numerical simulations are performed by integrating the generalized nonlinear Schrödinger equation by using the split-step Fourier method with a step $\sim 5 \mu \mathrm{m}$ [3].

The chosen HC-PCF geometry is a negative curvature fiber based on a single-ring structure surrounding by the hollow-core. This type of fiber generally supports a large family of high-order modes but in a specific condition ( $d=0.68 D$ with $d$ and $D$ the tube and core diameters $(150 \mu \mathrm{m})$, respectively), it can provide a single mode $\mathrm{LP}_{01}$ operation [4]. A schematic view is shown in the inset of Figure 1.a with our chosen parameters. The fiber is filled with a gas of Argon which have a nonlinear index equals to $n_{2}=4.86 \times 10^{-23} \mathrm{~m}^{2} / \mathrm{W}$ and the pressure is adjusted to obtain a zero-dispersion wavelength (ZDW) in the near infrared at $1170 \mathrm{~nm}$. The group velocity dispersion and the effective area $\left(A_{\text {eff }} \sim 992 \mu \mathrm{m}^{2}\right)$ are calculated from an analytical model [5] and included in the numerical simulations.

\section{Results and discussion}

The light is generated in the MIR from a cascade of nonlinear processes in the argon filled HC-PCF pumped by the two pump pulses. The initial pulses $U P_{1}$ and $U P_{2}$ have a duration of $180 \mathrm{fs}$ and $50 \mathrm{fs}$ at Full-Width at Half Maximum (FWHM) at the Fourier transform limit and the peak powers reach $63 \mathrm{MW}$ and $390 \mathrm{MW}$. The corresponding spectral bandwidth are $\Delta \lambda_{1} \sim 9 \mathrm{~nm}$ and $\Delta \lambda_{2} \sim 8 \mathrm{~nm}$ at FWHM. The group delay mismatch (GDM) between the two pulses is $\sim 176 \mathrm{fs} / \mathrm{m}$ and therefore, we consider a total fiber length of $50 \mathrm{~cm}$ to ensure a temporal overlap during the propagation. Figure 1 displays the total spectrum as a function of the fiber length. During the pulse propagation, the two pumps symmetrically undergo spectral broadenings due to the phase modulations and a 
portion of the pump energy enters in the anomalous dispersion regime at $\mathrm{z} \sim 10 \mathrm{~cm}$. In addition, the spectral tail of the broadened pulse $\mathrm{P}_{2}$ extends and overlaps with phase matched frequencies in which the energy transfer process takes place. Other spectral components are generated in the UV at $\sim 374 \mathrm{~nm}$ (zone 1), in the near-IR (zone 2) and MIR (zone 3) due to the interaction of the two main pulses with a large spectral bandwidth to achieve phase match processes mostly from a FWM.
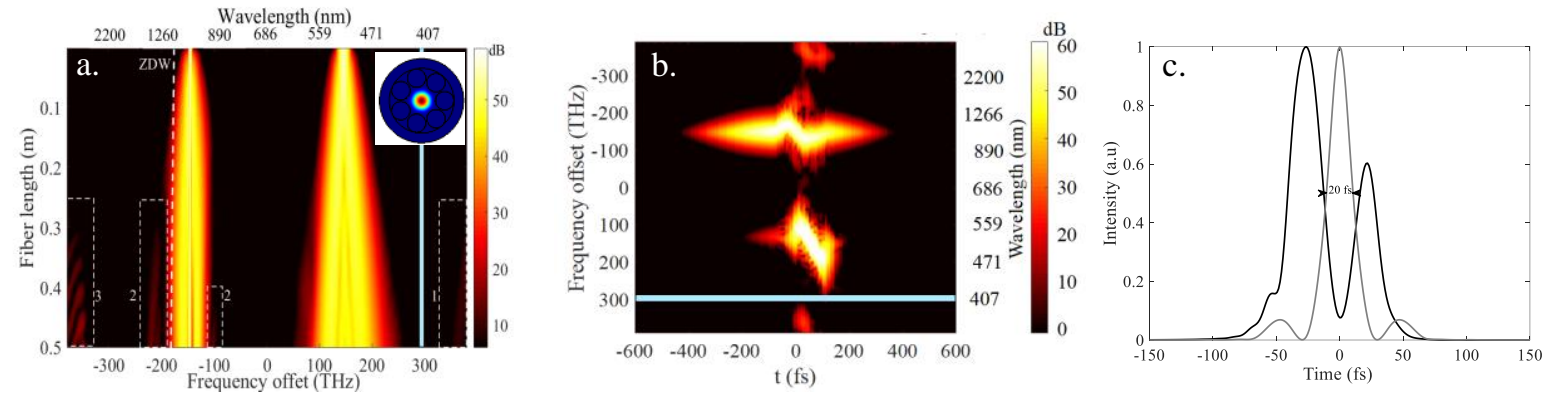

Figure 1. a. Total spectrum as a function of the fiber length with a logarithmic scale. b. Spectrogram at the HC-PCF output. The light-blue line represents the absorption band of the HC-PCF. c. Temporal profiles of the MIR band

(black solid-line). The grey solid-line corresponds to the pulse profile when the total phase is compensated.

As observed in Figure 1.a, this MIR band evolves during the propagation in the HC-PCF and their origins can be also discussed from the spectrogram (Figure 1.b) where we show the time-frequency distribution of the total field at the fiber output. During the propagation, the two pulses accumulate some temporal phase and therefore the instantaneous frequencies is quasi-linearly spread in time. As the spectral broadening of $U P_{l}$ is due to the interaction with $U P_{2}$, this chirp is only observed during the temporal overlap. Similarly, the additional bands (in the UV, NIR and MIR) can be generated only once the two pumps overlap. Each pulse owns a defined spectral bandwidth that evolves along the fiber (Figure 1a). From Figure 1.b, we can also observe that the MIR band has a spectro-temporal distribution. In fact, the pump and signal chirps are transferred to the idler at a specific time creating this spectrotemporal distribution.

At the output of the HC-PCF, the MIR band is selected with a flat-top bandpass filter and the pulse shape is directly calculated. By selecting the MIR band between 3 and $4 \mu \mathrm{m}$, the temporal shape exhibits two peaks separated by 48 fs. At the HC-PCF output, the accumulated phase can be compensated in order to reach an ultra-short pulse duration of $\sim 20$ fs. An energy of few nJ is obtained at the fiber output. Therefore, this scheme is highly desirable to develop a simple and robust pre-amplifier to seed subsequent ultra-fast amplifier in the MIR.

\section{Conclusion}

We numerically demonstrated that ultra-short pulses at 3 and $4 \mu \mathrm{m}$ can be generated through the interaction of a fundamental pulse and its harmonics in a HC-PCF filled with argon. The selected MIR band can directly provide sub-60 fs pulses and a duration of $\sim 20 \mathrm{fs}$ is expected if a system of phase compensation is used. The method is very promising to access the MIR spectral ranges in the ultra-short pulse regime in a robust fiber system.

\section{Acknowledgement}

ANR project (ANR-10-IDEX-03-02, ANR-17-EURE-0002,) and Bourgogne Franche-Comté council (SUM Project).

\section{References}

[1] R.I. Woodward, M.R. Majewski, D.D Hudson, S.D Jackson, APL Photonics 4, 020801 (2019).

[2] D.A. Koulouklidis, C. Gollner, V. Shumakova, V.Y. Fedorov, A. Pugžlys, A. Baltuška, S. Tzortzakis, Nat. Comm. 11, 292 (2020).

[3] C. Fourcade-Dutin, O. Zurita-Miranda, P. Mounaix, D. Bigourd, Fiber (2021). In press.

[4] P. Uebel, M.C. Günendi, M.H. Frosz, G. Ahmed, N.N. Edavalath, J.M. Ménard, P.S.J. Russell, Opt. Lett. 41, 1961 (2016).

[5] M.I Hasan, N. Akhmediev, W. Chang, J. Lightw. Technol. 36, 4060 (2018). 